\title{
Enquête
}

Archives de la revue Enquête

4 | 1996

La ville des sciences sociales

\section{La ville : cadre, objet, sujet}

Vingt ans de recherches françaises en histoire urbaine

The city : cadre, object, subject. Twenty years of french research in urban history

\section{Bernard Lepetit}

\section{(2) OpenEdition \\ Journals}

\section{Édition électronique}

URL : http://journals.openedition.org/enquete/663

DOI : 10.4000/enquete.663

ISSN : 1953-809X

\section{Éditeur :}

Cercom, Éditions Parenthèses

\section{Édition imprimée}

Date de publication : 1 novembre 1996

Pagination : 11-34

\section{Référence électronique}

Bernard Lepetit, «La ville : cadre, objet, sujet », Enquête [En ligne], 4 | 1996, mis en ligne le 11 juillet 2013, consulté le 19 avril 2019. URL : http://journals.openedition.org/enquete/663 ; DOI : 10.4000/ enquete.663

Ce document a été généré automatiquement le 19 avril 2019 


\title{
La ville : cadre, objet, sujet
}

\author{
Vingt ans de recherches françaises en histoire urbaine \\ The city : cadre, object, subject. Twenty years of french research in urban \\ history
}

\section{Bernard Lepetit}

\section{NOTE DE L'ÉDITEUR}

Bernard Lepetit devait contribuer à ce travail collectif par un texte intitulé « Le futur en doute. Catégories temporelles de la pratique urbaine ». Il entendait y poursuivre une réflexion conduite depuis une vingtaine d'années sur l'histoire urbaine et, plus largement, sur la ville. La rédaction de ce texte n'était pas encore commencée lorsque Bernard Lepetit nous a quittés.

L'article que nous publions ici, daté de juin 1991, ne présente certes pas l'état le plus récent de sa réflexion. Inédit en français - il est paru en 1992 dans la revue mexicaine Secuencia, Revista de Historia y Ciencias Sociales (24, p. 5-28) sous le titre « La historia urbana en Francia : veinte anos de investigaciones »-, il correspond toutefois de très près aux préoccupations de ce numéro, en décrivant les constructions successives dont la ville a fait l'objet chez les historiens français depuis le milieu des années soixante-dix. La réflexion de Bernard Lepetit l'avait conduit ces dernières années sur des voies différentes. Nous renvoyons les lecteurs désireux de poursuivre son itinéraire intellectuel aux indications bibliographiques que nous avons ajoutées à la fin de cet article.

1 L'un des premiers bilans de la recherche française en histoire urbaine était destiné à un public britannique. L'histoire urbaine anglaise, qui affichait dans les années soixante sa volonté de se constituer en discipline autonome, souhaitait disposer d'un état comparatif, peut-être pour mesurer le chemin qu'elle-même avait parcouru. J'imagine assez bien l'embarras de l'auteur pour cerner un domaine alors sans existence propre en France. Son constat fut très pessimiste. "If we consider the results achieved by urban history in France during the last half-century, we must admit that the crop is meagre and rather 
disappointing. Even today, this field of historical research is still in its infancy ${ }^{1}$.» Aujourd'hui, le paysage semble radicalement transformé: l'histoire urbaine est un chantier où l'on s'affaire. Les grandes thèses de doctorat depuis vingt ans s'accumulent ${ }^{2}$. La succession rapprochée des colloques, le nombre des participants qu'ils mobilisent, les publications fréquentes dont ils sont suivis témoignent de la même vitalité3. La collection intitulée "Histoire des villes », fondée en 1972, destinée à un public divers d'amateurs locaux et de professionnels de l'histoire, est un succès de librairie. Enfin, l'Histoire de la France urbaine, panorama en cinq volumes et plus de trois mille pages, est, à ma connaissance, sans équivalent à l'étranger ${ }^{4}$.

2 Pourtant, si l'on trouve, par exemple, des chaires d'histoire de l'Amérique latine ou d'histoire des relations internationales au sein des universités, on y chercherait vainement une chaire d'histoire des villes. Hors de l'Université, il n'existe pas plus d'institution de ralliement fortement structurée. Le Groupe de Travail international d'histoire urbaine, né en 1976 dans le cadre de la Maison des Sciences de l'Homme à Paris et où les chercheurs français ont toujours joué un rôle important, reste très informel. Aucune revue spécialisée ne peut davantage prétendre jouer le rôle de porte-drapeau ou d'espace d'échange intellectuel. Renvoyer cet état de fait à l'habituel individualisme des chercheurs est une explication trop courte : elle pourrait valoir pour tous les secteurs de l'histoire, même les mieux définis.

3 Comment alors, rendre compte de la situation de l'histoire urbaine en France ? Préciser l'orientation et le contenu des travaux qui en relèvent est nécessaire. Mais ce n'est sans doute pas suffisant pour comprendre un domaine neuf, à la fois foisonnant et peu structuré. Ses caractères présents et son avenir proche dépendent autant des conditions de sa formation, et de sa place dans la géographie du savoir. Ce seront là les principales étapes de l'analyse.

4 Avant de l'entreprendre, je dois indiquer ses limites. Le grand nombre des travaux et l'espace rédactionnel nécessairement restreint d'un article empêchent de tout citer. Ma propre spécialisation et une inévitable subjectivité conduisent à un certain arbitraire dans le choix. Les exemples relèveront presque toujours de ce qu'on nomme l'histoire moderne, du XVI ${ }^{\mathrm{e}}$ siècle au XVIII ${ }^{\mathrm{e}}$ siècle. Ces limites chronologiques ne déséquilibrent peutêtre pas trop le tableau : l'analyse de la ville préindustrielle a donné lieu à une bonne part du renouvellement de l'histoire urbaine.

\section{Les conditions de la formation de l'histoire urbaine}

Lorsqu'elles consacrent en 1970 un numéro spécial au thème " Histoire et urbanisation ", les Annales situent nettement l'entreprise : «Une fois de plus, c'est à un problème très actuel que nous avons voulu convier la réflexion historique. Le développement urbain mobilise depuis plusieurs années toutes les sciences humaines non seulement pour imaginer des projets d'aménagement, des remèdes à l'extraordinaire pathologie qu'il a engendrée, mais plus profondément pour prendre en main consciemment et scientifiquement ce qui semble être l'enjeu essentiel de nos sociétés. Dans ce concert prométhéen l'historien a, bien sûr, son mot à dire ${ }^{5}$." Tout ou presque est dit en une phrase : le social résumé dans l'urbain, le développement des villes et les désordres qui en résultent, l'aménagement nécessaire, la mobilisation générale des sciences humaines et l'intervention de l'histoire. Attente sociale, climat de réquisition scientifique non exempt 
de rivalités entre les disciplines: on s'efforcera de suggérer qu'à ces sollicitations externes s'ajoute une nécessité interne à l'histoire, liée à son évolution.

\section{La demande sociale}

6 La coïncidence entre les grands moments de développement de l'historiographie des villes et les périodes de crise urbaine a été souvent soulignée ${ }^{6}$. Du XVI ${ }^{e}$ siècle au XVIII ${ }^{\mathrm{e}}$ siècle, de l'Humanisme aux Lumières, congrégations religieuses et notables érudits multiplient les histoires de villes. L'exaltation de la civilisation citadine, la recherche systématique des signes de grandeur (vestiges archéologiques, grands hommes, fastes des périodes d'indépendance de la Cité) dans un passé qui cautionne en permanence le présent, constituent les thèmes essentiels de cette littérature. Son ambition est évidente. Dans une longue période d'atteinte à l'indépendance des villes, pendant laquelle les anciennes autonomies locales reculent devant le pouvoir royal, il s'agit de valoriser culturellement les vestiges d'une indépendance perdue. Pendant tout le XIX siècle, trois générations d'érudits locaux, animateurs de sociétés savantes, s'attachent à leur tour à rédiger des histoires urbaines. Elles manifestent une volonté d'enracinement dans le passé quand l'industrialisation vient rompre les équilibres anciens, provoquer la croissance des villes et remettre en cause les hiérarchies urbaines, quand le «cycle haussmannien » des aménagements s'en prend au tissu même de la ville. Elles affirment un patriotisme de clocher à un moment où, sous l'effet du développement des transports et de la délocalisation des investissements, s'unifie l'espace économique national.

7 La floraison historiographique actuelle s'inscrit aussi dans un mouvement de profonde mutation urbaine. Dans un pays resté longtemps plus campagnard que nombre de ses voisins, la mutation quantitative est considérable : en 1950, $55 \%$ des Français vivaient dans les villes; ils sont aujourd'hui $85 \%$. La dynamique de l'urbanisation généralisée prend des aspects de rattrapage, et la ville devient nécessairement l'objet d'une attention nouvelle. L'interrogation est d'autant plus anxieuse que la croissance s'accompagne de mutations qualitatives évidentes. Avec l'afflux de nouveaux habitants, les villes ont dû recomposer leur espace, affronter des problèmes sociaux aggravés, faire face aux multiples effets pervers du développement. L'attente sociale, face à un phénomène qui apparaitt inéluctable et incontrôlable, s'exprime à deux niveaux et de deux manières.

Le plus général est aussi le plus diffus. Dans une société française attachée à la terre, dont l'idéal urbain s'incarnait assez bien dans les petites villes ${ }^{7}$, le mouvement d'urbanisation rapide et, plus généralement, la croissance économique ont constitué un arrachement. En guise de compensation nostalgique se développe un désir d'enracinement. Le développement du phénomène de la « résidence secondaire » rurale, l'engouement pour la reconstitution généalogique familiale, l'intérêt porté à l'histoire d'un "monde que nous avons perdu » en sont autant d'exemples. La recherche urbaine s'inscrit dans ce courant, qui déborde donc largement les limites de la profession. La demande d'État a évidemment des raisons différentes : il s'agit ici de comprendre pour prévoir et organiser. L'importance de l'impulsion administrative dans le développement de la recherche urbaine a été grande. Des institutions telles que le Centre national de la recherche scientifique, la Délégation générale à la recherche scientifique et technique, ou la Direction de l'architecture et la Mission de la recherche urbaine du ministère de l'Urbanisme et du Logement dégagèrent des moyens financiers pour soutenir des équipes 
et mener à bien des programmes de recherche. On ne trouverait pas nécessairement un effort équivalent dans d'autres secteurs des sciences humaines.

9 Les historiens ne furent pas les premiers invités à constituer ces équipes. La foi technocratique dans les possibilités d'un aménagement fonctionnel, puis l'espoir de découvrir la théorie générale de l'urbanisation susceptible de régler la question urbaine l'emportèrent d'abord. L'échec conduisit à une double réorientation des enquêtes. D'une part, celles-ci se firent plus soucieuses des situations particulières de sociabilité et de la multiplicité des modes de vie locaux. D'autre part, elles manifestèrent souvent une volonté d'élargissement chronologique. Trois raisons militaient pour la prise en compte du temps :

10 1. La ville, dans les zones de fortes densités de peuplement et d'urbanisation ancienne d'Europe occidentale, est un phénomène de très longue durée. La faible malléabilité des formes urbaines s'ajoute à la permanence des sites pour assurer au passé une pesanteur maximum. La ville est « un conservatoire temporel ${ }^{8}$ ».

11 2. La ville est un phénomène total qui mêle tous les niveaux de la réalité : économique, social, politique, culturel. De ce fait, tout questionnement fractionné, qu'il soit le fait de la sociologie ou de la science économique par exemple, s'expose à manquer de pertinence. $\mathrm{Au}$ contraire, une lecture totalisante, comme affirme l'être l'analyse historique, risque de mieux prendre en compte l'effet des inter-relations.

12 3. Deux traditions parcourent l'urbanisme: un modèle progressiste nourri de logique fonctionnelle, et un autre, culturaliste, soucieux de retrouver l'ancienne unité organique de la Cité. . La perception des limites d'un aménagement technocratique incapable de résoudre les problèmes urbains ne pouvait manquer de valoriser le second courant. Le passé, alors, garde le témoignage de modèles réussis, fussent-ils de l'ordre du mythe : aux historiens de les redécouvrir.

\section{La géographie du savoir}

13 Tardivement développée, l'histoire urbaine se trouve confrontée aux analyses menées par les sciences sociales voisines. Les géographes, les économistes, les sociologues, les architectes, les philosophes et d'autres encore ont eu leur mot à dire sur la ville. Elle se trouve ainsi ouverte aux quatre vents de la recherche et confrontée aux grands courants qui l'animent. Pour la décrire, il faut aussi prendre la mesure de ce champ de force.

14 L'étude historique des fonctions citadines s'est d'abord nourrie des méthodes et des réflexions des géographes. Développées dans le cadre de recherches universitaires ou de rapports destinés à guider l'action des organismes d'aménagement du territoire, ces analyses développaient une démarche en deux temps pour comprendre la croissance des villes et les configurations de l'armature urbaine. Il s'agissait de classer d'abord les villes selon le niveau et le type des fonctions que chacune exerçait, puis de proposer à l'aide de combinatoires diverses des typologies et des hiérarchies urbaines ${ }^{10}$. Trois raisons au moins facilitèrent l'introduction en histoire d'interrogations de cette nature: une communauté fréquente de formation puisque l'histoire et la géographie constituèrent longtemps des disciplines associées dans les cursus universitaires, une approche descriptive des activités économiques, une grande attention de la majorité de ces études géographiques à un passé dans lequel le présent trouvait son explication. Au-delà de la dimension descriptive des fonctions urbaines, les historiens en retinrent une série de 
questions sur le mode de fonctionnement économique des villes et sur les caractères originaux des armatures urbaines de l'époque préindustrielle.

Concernant les formes urbaines, trois modèles de lecture se proposaient à l'historien parallèlement à la sienne propre. Le premier, d'inspiration marxiste, se développait en réaction aux certitudes des aménageurs : « au fait urbain » succédait la « question urbaine ${ }^{11}$ \#. Là où les planificateurs voyaient dans les formes prises par le développement des villes un désordre, auquel une politique adéquate remédierait, les néo-marxistes y virent au contraire l'expression d'un ordre, celui du capital, servi par une politique, celle de l'État. Les disparités liées à la croissance, alors, ne sont plus accidentelles mais sont les conséquences de déterminants économiques et politiques qu'il s'agit de mettre au jour ${ }^{12}$. L'influence de ce courant de pensée sur l'histoire urbaine est difficile à apprécier. On peut sans difficulté déceler des orientations communes: le souci d'insérer l'urbain dans la société englobante, ou l'analyse de la construction de l'espace citadin en termes d'enjeu social.

Mais inversement, l'attention portée par le néo-marxisme aux sociétés capitalistes développées, l'absolue priorité qu'il donne à l'élaboration théorique sur l'observation empirique, la dissolution qu'il opère finalement de la question urbaine dans la question sociale - dissolution qui empêche de penser l'autonomie de l'urbain et la spécificité du domaine de recherche - constituent beaucoup d'obstacles à la communication avec les historiens. Ces derniers n'ont sans doute trouvé dans l'ensemble de ces travaux que ce que l'imprégnation marxiste diffuse de l'histoire française leur y faisait chercher.

Beaucoup plus directe était l'interpellation venue d'un groupe d'architectes et d'urbanistes, et d'un courant qui s'alimentait à la pensée de Michel Foucault. Ici, au moment où l'histoire se faisait urbaine, la recherche urbaine se faisait historique. La part de ce qui revient à l'histoire urbaine stricto sensu devient difficile à apprécier dans des recherches souvent menées au même moment. Des travaux conduits en particulier dans le cadre de l'Institut français d'architecture, on peut dégager des interrogations majeures ${ }^{13}$ : que peut dire un projet urbain sur la société dans laquelle il est produit? Quels rapports s'établissent entre les représentations mentales, les plans d'aménagement et les opérations réalisées? Quelles sont les contraintes formelles héritées du passé qui pèsent sur l'aménagement urbain? Comment une société investit-elle le bâti pour lui donner un sens nouveau? L'attention au répétitif plus qu'à l'exceptionnel, à l'habité plus qu'au monumental, à la diversité du vécu derrière les règles urbanistiques, à la dialectique passé-présent (un présent constamment soumis aux pesanteurs du passé, mais le réinvestissant en permanence de valeurs nouvelles) constituent autant de titres d'intérêts pour la corporation historienne au sein de laquelle se développent des interrogations semblables.

18 La logique du recours à l'histoire naît pour la recherche architecturale de l'objet même de l'analyse: la ville a une épaisseur temporelle. Pour Michel Foucault, la raison de l'attention au passé est autre. Elle trouve son origine dans un fonctionnalisme revisité. Historiquement, essentiellement aux XVIII ${ }^{e}$ et $x x^{e}$ siècles, la ville est pour lui le lieu et le moyen d'un renforcement du pouvoir. À travers les réflexions du philosophe et des équipes qu'il anime ou inspire, la ville apparait comme le lieu privilégié de la mise en place des méthodes de la disciplinarisation des classes inférieures. Cette mise en place s'opère, pour le plus grand bien de l'ordre social et de la productivité du travail, au bénéfice des classes dirigeantes. Il s'agit d'une vision assez instrumentale de la ville : c'est en effet à la fois la multiplication des équipements de contrôle social (prisons, hôpitaux) 
et la mise au clair de l'espace public (diffusion de l'éclairage des rues, numérotation des maisons) qui concourent à cet assujettissement plus efficace ${ }^{14}$. D'espace de liberté, la ville devient instrument de pouvoir. Cette mutation des fonctions urbaines, moins aisément mesurable que celle des géographes ou des économistes, est précisément datable. Ainsi, c'est le rôle historique de la ville, organisée et disciplinaire, qui conduit M. Foucault à rencontrer les historiens sur leur terrain. Le peuple et les polices, objets d'une attention renouvelée, se rencontrent dans l'espace citadin; voici à nouveau liés rapports sociaux, politiques urbaines et organisation du territoire.

\section{L'évolution de la discipline historique}

19 Le mimétisme, ou l'air du temps, ne suffisent pas à eux seuls à expliquer l'attention de l'histoire pour l'objet urbain. Des raisons doivent également être trouvées à l'intérieur même de la discipline. On constatera d'abord que l'histoire urbaine s'intègre parfaitement dans cette «nouvelle histoire» dont la diffusion de l'influence de l'école des Annales a presque généralisé la pratique. «Pour en cerner l'ambition et le développement », le plus simple « est de tenir ensemble deux objectifs et deux idées : que l'histoire s'enrichisse en sujets et en méthodes par le recours aux disciplines voisines et même par l'éclatement provisoire des cloisonnements disciplinaires; qu'elle reste pourtant un savoir global, œcuménique, réunissant les conditions d'intelligibilité maximale des phénomènes sociaux ${ }^{15}$ ». Recours aux disciplines voisines, effacement provisoire des cloisonnements? Il suffit de consulter la table des matières des toutes premières grandes thèses d'histoire urbaine pour constater que l'ambition s'en dessine : conditions démographiques, activités économiques, société urbaine, vie religieuse et pouvoir ici; l'espace et les hommes, l'économie et ses rythmes, le style de vie là ; démographie, structures sociales, structures mentales et comportements collectifs ailleurs encore.

On verra là une autre raison de son succès. À l'heure où l'ambition d'abord proclamée d'une histoire totale recule pour devenir un horizon de recherche, ou même s'efface totalement, le développement de l'histoire urbaine peut apparaître comme un remède palliatif. Voici en effet un objet d'étude, la ville, qui « rassemble toutes les questions que pose l'évolution pluriséculaire du système de civilisation ${ }^{16}$ ». Comment ne pas espérer de sa description, ou mieux de sa compréhension, une nouvelle approche de la globalité ? Si toute l'histoire est dans l'urbain, comprendre la ville devient un moyen de reconquérir le paradis perdu et d'accéder à un savoir total renouvelé.

On avancera une autre hypothèse encore, moins globalisante, qu'on illustrera sur un exemple, celui de l'évolution de l'histoire sociale en France. Pendant une quinzaine d'années, le débat y a tourné autour d'une question: quelles sont les catégories pertinentes pour comprendre l'organisation d'une société, et particulièrement de la société préindustrielle? Les premiers, Ernest Labrousse et ses élèves proposèrent de définir les structures sociales en termes de statut socio-professionnel (à la manière des codes élaborés par l'Institut national de la statistique et des études économiques pour la France d'aujourd'hui) et de hiérarchie des fortunes (mesurée grâce à une analyse sérielle des archives fiscales et notariales). La principale opposition vint d'un groupe d'historiens animés par Roland Mousnier. Pour eux, la société d'Ancien Régime n'est pas une société de classes, mais d'ordres. Aussi, la hiérarchie sociale ne peut pas se déduire d'une échelle des fortunes ou de la place de chacun dans les rapports de production. Elle repose au contraire sur l'estime sociale collective attachée à chaque état ou à chaque fonction, et le 
seul découpage acceptable est celui qui rassemble les groupes qui marient entre eux leurs enfants.

Entre ces a priori irréductibles, aucun dialogue n'était évidemment possible. Après une phase de mobilisation de part et d'autre, aboutissant à de beaux travaux (dont les premières thèses d'histoire urbaine), l'impasse apparut totale et stérilisante. Pour relancer la recherche, il fallait abandonner ce terrain d'un combat sans issue. Jean-Claude Perrot apporta les premières justifications d'une telle attitude dans un article au titre révélateur : «Rapports sociaux et villes ». Trois propositions enchaînées s'y lisent: les études structurelles, si elles constituent une fin en elles-mêmes, enferment l'histoire dans un débat dogmatique; l'analyse des relations sociales, et non plus des structures, constitue le moyen de surmonter la stérilité de ce débat ; l'étude des faits urbains est une manière privilégiée de mettre au jour ces relations car l'espace urbain «consacre des rapports sociaux ${ }^{17} »$. Voici l'histoire urbaine désignée comme la voie pour sortir d'une impasse historiographique spécifique en surmontant les blocages de l'histoire sociale.

\section{La nouvelle histoire urbaine}

\section{L'invention de l'urbain}

Pour préciser la mutation qualitative qui a accompagné le développement quantitatif de l'histoire urbaine, deux textes publiés à quinze ans d'intervalle suffiront sans doute. Leur caractère très révélateur autorise peut-être à les citer longuement. Voici d'abord comment Pierre Deyon, en 1967, justifiait l'une des premières monographies urbaines achevées en France à une date récente. "Notre connaissance du XVII e siècle comporte bien des ombres; plus d'un problème demeure en suspens, suscitant la curiosité et les discussions des historiens. L'étude minutieuse de la société à l'époque classique apparaît comme une des conditions nécessaires à la poursuite féconde de ces débats ; l'essai qui va suivre voudrait être une contribution à cette entreprise collective. Le cadre local imposait des limites, mais seul il permettait de mesurer, de compter, de procéder à des rapprochements incessants entre les divers plans de la réalité historique et peut-être de découvrir les relations nouvelles que d'autres études pourraient reprendre et vérifier. Amiens, capitale provinciale : c'est l'état des sources qui, pour une très large part, a guidé ce choix géographique. Important centre de commerce et de fabrication textile, Amiens ne constituait pas, au point de vue économique, un observatoire négligeable. En tout cas, elle offrait un cadre commode pour une étude des promotions et des différenciations bourgeoises ${ }^{18}$.»

Très clairement à ce moment, l'histoire urbaine apparaît comme un moyen, non comme une fin. Trouvant sa justification à l'extérieur d'elle-même, elle n'a pas véritablement acquis son autonomie. Elle s'inscrit dans une histoire sociale qui l'englobe, et se donne pour ambition de contribuer aux débats qui l'animent. Pourquoi la ville alors? Essentiellement parce qu'elle constitue « un cadre commode », un observatoire privilégié. Monde des contrastes sociaux, où les hiérarchies s'affirment de manière plus structurée et plus fine à la fois, elle fournit un champ privilégié pour les controverses de l'histoire sociale. Lieu d'exercice d'un pouvoir administratif qui se livre au contrôle quantifié des hommes et des produits, elle est site d'accumulation de l'archive. Elle est enfin le microcosme où, tous les plans de la réalité se rapprochant, des relations explicatives ailleurs inaperçues car trop distendues se donnent à lire. 
25 En 1983, l'introduction de la thèse de Jean-Pierre Bardet permet de mesurer le déplacement des problématiques. "L'existence d'une ville constitue un phénomène pratiquement inexplicable, parce qu'en Europe, l'urbain s'inscrit dans la très longue durée. En revanche, la dynamique propre d'une cité peut être intelligible. Le décodage de l'urbanisation ne consiste pas seulement à saisir les moteurs de la reproduction citadine inextricablement liés au mode de production, il amène aussi à qualifier et à comprendre l'effet transformateur de la vie urbaine. La ville est objet et sujet. Construite par les hommes, elle est la plate-forme de leurs activités. En même temps, les hommes et les femmes qui façonnent la ville se soumettent à ses lois. Elle détermine une pensée, des comportements et une écologie qui à leur tour participent aux modalités du renouvellement urbain. C'est cet échange complexe que je voudrais éclairer ${ }^{19}$. $»$ La ville acquiert ainsi une double autonomie. Elle se trouve placée au centre des interrogations : quels sont les moteurs de l'urbanisation, quelles sont les capacités transformatrices de l'urbain? Au plan de la connaissance elle est en tant que telle devenue objet de l'histoire. Cette spécificité du mode d'appréhension redouble, en y trouvant sa justification, celle du réel. Objet - construit par les hommes, analysé par la science historique - la ville est aussi sujet de l'histoire. Elle est plus qu'un cadre, plus que la simple somme des éléments qui la composent. Par son existence même, elle est créatrice d'innovations qui affectent son propre devenir.

26 Il est facile de dater la mutation qui fait passer la ville du statut de sous-produit de recherche obéissant à des préoccupations thématiques autres à celui de personnage central. Un article de 1974, dont l'ambition dépassait la dimension historiographique seule avouée, et la thèse de Jean-Claude Perrot, achevée en 1973 et publiée en 1975, constituent les textes charnières ${ }^{20}$. En affirmant que le sujet de l'histoire urbaine existe et qu'il y a une unité et une créativité des phénomènes citadins, ces textes fondaient du même coup un domaine de recherches spécifiques.

\section{Une expérience exemplaire}

27 Une étude plus précise du livre de Jean-Claude Perrot permettra de mieux en dégager les caractères et les difficultés. Apparemment, à consulter d'abord les titres des chapitres, le livre s'inscrit dans l'un des courants dominants de l'historiographie française, placé depuis le début des années cinquante sous l'influence d'Ernest Labrousse, et qui met en œuvre une histoire quantitative attentive à dégager les mouvements alternés de la conjoncture, des structures économiques et sociales de longue durée. Pourtant, à la différence de toutes les monographies d'histoire urbaine du moment, le livre ne comporte aucune étude particulière des «structures sociales ». La critique du débat sur la nature des sociétés d'Ancien Régime, que Jean-Claude Perrot avait publiée quelques années auparavant, conduisait évidemment à y renoncer. Mais la solution qu'il adopte en 1975 est différente de celle qu'il suggérait sept ans plus tôt. En 1968, considérant que l'analyse des structures était nécessairement tautologique, il préconisait d'étudier les relations sociales. Les cérémonies publiques, les formes associatives, les lieux de rencontre, les manifestations de la violence constituaient autant de dimensions d'une sociabilité citadine dont la description permettrait d'accéder à la connaissance des sociétés du passé. Le quartier, la rue, l'atelier, le cabaret constituaient les lieux où se révélaient les façons d'être ensemble, d'ailleurs plus souvent populaires que bourgeoises ou aristocratiques ${ }^{21}$. 
Mais précisément, tous ces lieux ne constituaient encore qu'un cadre, observatoires privilégiés qui favorisaient à la fois la rencontre et son contrôle.

En 1975, Jean-Claude Perrot tient pour réductrice l'attitude qui consiste à croire qu'on a compris une société après avoir décrit les groupes qui la composent et leurs relations. «Une lecture perspicace doit sentir que les comportements de la population, la pratique médicale, les processus qui règlent la production, les échanges, l'aménagement des quartiers décrivent efficacement les fondements de l'histoire sociale» (p. 944). La ville, ainsi, n'a pas de nature propre : elle relève tout entière du social dont elle est comme une « scorie » (p. 947), comme un résidu provenant du refroidissement superficiel des coulées de lave, au sens où l'entendent les vulcanologues. Il en naît une conséquence méthodologique. La simple juxtaposition de plusieurs études (démographie, économie, société, culture) aboutirait à faire tomber le sujet en ruine : «La première démarche consistait donc à croiser les sources entre elles puisqu'on admet qu'elles parlent de la même chose " (p. 947), c'est-à-dire de la ville moderne. Ainsi, l'historien ne peut pas confondre les structures de la réalité passée avec le découpage des séries d'archives; la primauté de l'évidence de l'archive est remise en cause au profit de l'identification et de la construction d'un objet.

Le livre, en ce sens, est un modèle, une "copie théorique", c'est-à-dire une représentation simplifiée et abstraite de la réalité qu'il envisage, construite à partir d'un système de relations qui répond à des principes de cohérence interne et n'entre pas en contradiction avec les données empiriques disponibles. Le premier impératif de la recherche est donc d'établir, en se développant, que son objet existe au moment où elle l'étudie. Caen, au XVIII ${ }^{e}$ siècle, devient une "ville moderne ", c'est-à-dire, pour reprendre la définition du dictionnaire, appartient au temps de celui qui parle, l'historien du $\mathrm{xx}^{\mathrm{e}}$ siècle. Deux traits définissent ici la modernité urbaine: le passage d'une économie stationnaire à une économie de mouvement d'une part, l'invention du fonctionnalisme de l'autre. Le premier trait conduit à ne plus réduire, comme le fait trop souvent l'histoire sérielle, le changement à sa mesure quantitative. « La taille de Caen, le produit global, s'ils étaient mieux connus, resteraient muets sur cette révolution silencieuse qui la hausse au degré d'urbanisation où se trouvent déjà, passé Paris, quatre grands ports et quelques places fameuses de l'intérieur » (p.951). Le second trait conduit à mettre en évidence, dans la pensée et la pratique des citadins, à partir du milieu du XVIII ${ }^{e}$ siècle, la série des principes liés qui définissent le fonctionnalisme : le système socio-économique doit, pour être compris, être décomposé en fonctions distinctes; il existe entre chacune de ces fonctions des relations, des co-variations (richesse et mortalité, niveau de population et commerce, maladies et activités professionnelles); il existe entre chacune des fonctions et les espaces où elles s'exercent une relation simple (ce qui justifie bien entendu l'intervention sur l'espace pour produire une modification de fonction). L'un et l'autre trait amènent à disqualifier le lien auparavant établi entre l'industrialisation et l'urbanisation, entre la prolétarisation $\mathrm{du} \mathrm{xIX}^{\mathrm{e}}$ siècle et les problèmes urbains: " "L'invention" de la question urbaine surplombe d'un siècle celle de la question sociale " (p. 9).

La modernité de l'objet porte avec elle deux conséquences. Elle conduit d'abord à accorder une attention particulière aux représentations que le passé se donne de luimême. Le discours qu'une société tient sur elle-même ou que ses pratiques révèlent informe à la fois le monde dans lequel elle vit (dans le premier sens en français du verbe informer : donner à la fois une signification et une forme) et l'historien qui l'étudie (dans 
le second sens d'informer: mettre au courant). Pourtant, notre expérience quotidienne nous alerte : les sociétés sont pour partie aveugles à leur propre nature et multiplient les ruses interprétatives.

Mais les pratiques qui découlent de cette cécité partielle - qu'il s'agisse des comportements démographiques, de l'aménagement du territoire ou des conflits entre les corporations de métiers - ont leur efficace propre, et il est toujours possible de considérer les propositions des observateurs passées comme un ensemble d'hypothèses à confronter aux données empiriques. Seconde conséquence de la modernité de la ville du XVIII ${ }^{\mathrm{e}}$ siècle : le chercheur est installé dans une considérable proximité avec son objet. En apparence, il s'agit d'un avantage : s'il est relativement facile de trouver dans la documentation du XVIII ${ }^{e}$ siècle des réponses aux questions que l'historien $\mathrm{du} \mathrm{xx}^{\mathrm{e}}$ siècle pose, c'est d'abord parce que les questions des deux époques à propos de la ville sont les mêmes. Mais cette facilité est un piège : la proximité rend difficile, sinon impossible, la remise en cause critique de l'analyse fonctionnelle qui appartient elle-même à la classe des idéologies; toute une part de l'analyse urbaine d'aujourd'hui en reste imprégnée. «Connaître le flou de nos propres lunettes» (p.11), assurer un "va-et-vient continuel entre les techniques de maintenant et l'horizon des Lumières" (p.13), jouer de la distance temporelle et de l'épaisseur herméneutique qui nous séparent $d u \mathrm{XVIII}^{\mathrm{e}}$ siècle: tels sont les moyens utilisés pour organiser une tension créatrice de sens et compréhensive entre la ville d'hier et les catégories qui la donnent à voir.

Mais en affirmant que « tout le sens est dans le rapport entre [les] différents niveaux » de l'organisation sociale, Jean-Claude Perrot donnait aux historiens des villes un objectif particulièrement intimidant ${ }^{22}$. Si la saisie séparée des différents niveaux de la réalité semble n'opposer que des problèmes traditionnels, liés à l'insuffisance des sources, comment appréhender les rapports entre ces niveaux ? Comment étudier ce phénomène socio-culturel total où les risques de rencontrer des causalités circulaires abondent? Le degré de complexité auquel était située l'ambition historiographique comportait en luimême des risques pour l'entreprise. Deux phrases, qui définissent le caractère de la nouvelle histoire urbaine peuvent servir de révélateur : «La ville devient le personnage central de toute une génération de travaux", écrivaient en 1974 L. Bergeron et M. Roncayolo ; en 1983 F. Bedarida soulignait : "The many fields of research have one great common factor : refusal to consider the city as merely inert, lifeless ${ }^{23}$ \#. L'ambiguïté des métaphores biologiques dans le domaine social a déjà été soulignée et il n'est pas question ici d'instruire un mauvais procès. Le but de la nouvelle histoire urbaine n'était pas de doter la ville d'une quasi-intentionnalité, mais de souligner à la fois l'autonomie de l'urbain et son extraordinaire complication.

On voit bien les dangers qu'une telle globalisation implique. D'une part, bien peu de chose, même à l'époque préindustrielle, ne touchent pas par un côté ou un autre à la ville. Si rien de ce qui est urbain n'échappe à l'histoire urbaine, la dilatation et la diversité du champ de recherche deviennent extrêmes. En même temps, comment croire qu'on enserrera aisément dans un réseau de définitions l'ensemble des fils qui forment la toile urbaine? Faute d'une réflexion suffisante sur la nature de ces liens et sur les médiations qui autorisent à passer d'un niveau à l'autre de la réalité, ou d'un inventaire des phénomènes cumulatifs et des effets de blocage nés de la ville, le risque existe de produire un discours dont le rôle essentiel consisterait à maintenir l'unité artificielle d'un espace de recherche infiniment distendu. Mais à l'inverse, si l'espace citadin n'est que la simple projection d'une société sur la carte, la simple traduction au sol d'inégalités sociales, la 
ville n'est qu'une ombre de la caverne. Si l'analyse des relations sociales se réduit à saisir la manière dont les hommes se "rencontrent» dans les villes, alors l'histoire urbaine n'est qu'un voile destiné à masquer l'incapacité provisoire de l'histoire sociale à fournir des explications des solidarités et des conflits qui soient issus de la construction même des sociétés, et la ville une fois de plus n'est qu'un théâtre.

\section{Changements d'échelle}

d'échapper aux deux risques symétriques d'une complexification opacifiante et d'un évanouissement de l'objet. Elle suppose explicitées des questions qui aient pour but de cerner les spécificités de l'urbain. Elle suppose, pour reprendre le titre d'un article aux ambitions plus étroitement circonscrites, de comprendre «les villes comme systèmes dans un système de villes ${ }^{24}$ ». Selon le sens qu'on donnera au mot système, la recherche peut s'orienter dans plusieurs directions. Si l'on définit le système urbain comme celui qui organise en une configuration spatiale et hiérarchique un ensemble de villes, alors l'étude est celle des réseaux et des armatures, étudiés dans leur relation à l'espace. Si l'on définit la ville comme « une formation réelle qui s'inscrit sur le sol dans une topographie particulière, qui s'imprime dans une société structurée, qui s'exprime par des institutions et se traduit par un genre de vie et une culture ${ }^{25}$ ", alors la recherche se développe en analyse systémique de la «formation » considérée. Dans les deux cas, mais dans des sens différents, il s'agit d'un changement d'échelle par rapport aux monographies de villes qui ont servi de cadre au premier renouvellement de l'histoire urbaine. Je voudrais le suggérer sur deux exemples.

Fernand Braudel le soulignait dès le milieu des années soixante : jamais une ville ne se présente sans l'accompagnement d'autres villes, plus grandes ou plus petites, proches ou lointaines, points d'appui, relais ou rivales ${ }^{26}$. Dans une économie préindustrielle, l'existence de systèmes urbains n'est pas une hypothèse forte. Une ville ne peut pas se concevoir sans l'existence d'un espace qui l'environne et d'où elle tire l'essentiel de sa subsistance (ni les grains, ni le bois, produits lourds, ne voyagent, sauf exception, sur de très longues distances), une bonne part de sa population (les balances démographiques naturelles des villes sont négatives) et de ses revenus (puisque les plus riches des citadins sont des propriétaires fonciers). Même si les villes n'entretenaient aucune relation entre elles, elles n'en seraient pas moins dans la dépendance les unes des autres : l'absence de révolution agricole jusqu'au XIX ${ }^{\mathrm{e}}$ siècle s'oppose à ce que l'ampleur des prélèvements possibles en denrées, en rentes et en hommes soit indéfinie. La localisation, la taille et le rythme de croissance de chaque ville dépendent ainsi de celle des autres. Poser une question d'histoire urbaine, c'est se demander comment un tel système est à l'origine de son propre devenir.

Si l'on considère par exemple la France des années 1830, avant le développement d'une industrialisation d'un nouveau type, de nombreux indices (tels que l'évolution de la géographie des prix ou les modalités de la diffusion des innovations) montrent un fonctionnement régional différent du système urbain ${ }^{27}$. Au nord, l'unification de l'espace économique, l'intensité des liaisons interurbaines, la réceptivité des sociétés citadines caractérisent un fonctionnement du système urbain en réseau. Au sud, la persistance d'un cloisonnement économique, le retard à innover, la moindre intensité des flux de relation, le repli plus important des sociétés urbaines sur elles-mêmes désignent un 
fonctionnement du système urbain en armature qui juxtapose des cellules (formées chacune d'une modeste métropole locale et de son arrière-pays) peu reliées entre elles. Une première explication renvoie cette différence à l'histoire de la mise en place du réseau routier qui débute dans les années 1740 et profite davantage à la moitié nord du pays qu'à la moitié sud ${ }^{28}$. Tout dans les caractéristiques de l'édification des routes prouve l'influence du système urbain. Sous l'Ancien Régime, les routes construites renforcent l'armature urbaine la plus ancienne, accentuent le poids de Paris (et du Bassin parisien), desservent en priorité les villes caractérisées à la fois par l'exercice de la fonction administrative et de la fortune foncière. La même reproduction d'une structure par une autre se retrouve après la Révolution. En 1811, l'administration des Ponts et Chaussées établit le classement des routes impériales; ce classement est aussi un programme d'équipement, car le réseau n'est pas achevé à cette date et le classement d'une route détermine l'importance des financements qu'elle reçoit. Il calque à son tour le réseau des routes sur la nouvelle armature administrative du pays : à taille égale, les nouveaux chefs-lieux bénéficient d'une meilleure desserte que les autres villes.

Peut-on dire alors que le système urbain des années 1830 trouve son origine dans la création des départements, puis dans le choix des préfectures et des sous-préfectures qui en forment les centres ? La réalité est plus complexe. Malgré une mobilisation très active des villes au moment de la réforme ${ }^{29}$ qui renvoie bien entendu aux équilibres d'avant 1789, la nouvelle carte administrative s'inscrit en rupture par rapport à l'Ancien Régime : les villes promues ou déclassées dans l'ordre administratif ont été nombreuses. Dans l'ordre hiérarchique, le nouvel équilibre entre les villes apparu au début de la Révolution et qui favorise les villes petites et moyennes au détriment des métropoles régionales se trouve structuré pour longtemps : jusqu'à la Seconde Guerre mondiale, la France est un pays de petites villes. Dans l'ordre spatial, l'armature des chefs-lieux développe sur tout le territoire une grille uniformisatrice et tend à fossiliser cette organisation régulière. Mais dans l'ordre économique, ce sont des fonctionnements anciens que la réforme administrative généralise : chaque chef-lieu devient un point de rassemblement pour les rentiers de la terre et pour les membres des professions libérales (notaires, avocats, médecins) qui développent dans leurs comportements, leurs modes de vie et leurs habitudes de consommation des modèles propres aux milieux privilégiés (noblesse du haut clergé) du xviII ${ }^{e}$ siècle. Ils donnent ainsi à ces villes certaines de leurs fonctions économiques les plus durables en faisant d'elles des centres d'activités de consommation 30 .

Ces deux formes nouvelles d'aménagement du territoire (la route et l'administration territoriale) permettent de souligner la manière complexe dont le système urbain participe à son propre devenir, dont il est à la fois, pour utiliser le vocabulaire de la statistique, une variable explicative et une variable expliquée. Cette complexité tient à ce que dans l'espace les formes géographiques, les équipements structurants et les fonctionnements économiques ne coïncident jamais exactement. Elle tient à ce que l'ancien et la nouveauté ne s'enchaînent jamais de manière linéaire. La route, équipement structurant nouveau, renforce les anciennes configurations géographiques en desservant d'abord, et mieux, les villes les plus importantes. Mais, dans le même temps, elle subvertit cette géographie en dotant d'un contenu relationnel nouveau les formes passées de l'organisation du territoire, et en permettant un fonctionnement plus progressiste de l'espace économique. La carte administrative mise en place sous la Révolution est également une structure nouvelle. Elle s'inscrit en rupture par rapport aux cartes 
anciennes, établit de nouvelles hiérarchies et projette sur le territoire de nouvelles distributions spatiales. Mais celles-ci, au contraire, sont porteuses de modes anciens de fonctionnement économique des systèmes urbains (un ensemble de minuscules capitales locales) et viennent assurer sa diffusion dans l'espace français et sa persistance dans le temps, au-delà même de la révolution industrielle. Ainsi, en produisant du neuf avec le vieux et du vieux avec le neuf, le système urbain est en permanence dans un déséquilibre partiel. Il est à tout instant engagé dans un processus historique de transformation de luimême.

Pour autant, son évolution n'est ni mécanique ni aisément prévisible. Les formes nouvelles de structuration de l'espace se développent selon des temporalités différentes. Elles s'appliquent d'autre part à des régions dont les passés diffèrent. En France, les systèmes d'habitat et les formes d'urbanisation sont l'image peu déformée de très anciens schémas, sans doute plus que millénaires, de peuplement. L'inégale densité des trames administratives d'Ancien Régime et la domination parisienne sont le produit de l'histoire de la construction de l'État depuis le Moyen Âge. Les formes des réseaux commerciaux, la densité relative des foires épisodiques, des marchés hebdomadaires et des boutiques permanentes renvoient sans doute aux deux temporalités précédentes. Comme les derniers livres de Braudel l'ont suggéré, les géographies anciennes, «ces réalités entassées les unes sur les autres", sont constamment reprises par les hommes à des échelles différentes dans des constructions nouvelles de l'espace ${ }^{31}$.

Changeons maintenant d'échelle pour considérer les sociétés urbaines. Ce sont, on le sait, des sociétés mêlées. La croissance et même simplement le maintien du niveau de leur population supposent l'existence de flux migratoires permanents. L'importance du nombre des nouveaux venus et l'étendue du bassin migratoire où ils se rencontrent dépendent de la taille de la ville considérée, de sa fonction et de la conjoncture économique. Orientés en sens contraire, les flux de sortie sont toujours importants même s'ils sont plus difficiles à saisir. Mais, quoi qu'il en soit, la ville est un vaste carrefour où se mêlent populations stables et populations mobiles aux parcours et aux projets variés. Même en l'absence de toute autre cause, par simple nécessité démographique, les sociétés urbaines sont des sociétés plurielles où la question des identités et des identifications se pose d'une autre manière que dans les sociétés villageoises plus enracinées. L'historiographie, traditionnellement, abordait la question de deux manières. D'une part, on l'a vu, elle projetait sur les sociétés urbaines du passé des catégories analytiques qui trouvaient en elles-mêmes leur fin et leur commencement. L'historien pouvait utiliser les grilles et la rhétorique d'une sociologie classificatrice ${ }^{32}$, ou préférer interpréter le langage des acteurs dans une démarche herméneutique empruntée à l'anthropologie culturelle inspirée de Clifford Geertz ${ }^{33}$. Il pouvait préférer les classes, les ordres ou les catégories socio-professionnelles comme principe explicatif des sociétés anciennes. Dans tous les cas, la prédétermination de la démarche et du classement résolvait d'un coup la question des identités sociales. D'autre part, l'historiographie traditionnelle tendait à considérer les populations stables des villes comme seules porteuses d'une «identité citadine » que des mesures de contrôle actif des flux d'immigrants et une politique d'intégration très restrictive préservaient, et appliquait aux populations migrantes le concept d'acculturation ${ }^{34}$. C'était ne tenir compte ni de la variété des trajectoires et des projets individuels et familiaux, ni de la multiplicité des structures d'identification possible, ni de la complexité des conjonctures et des situations où s'actualisent les identités diverses des populations urbaines ${ }^{35}$. 
41 On se souvient des premiers mots de la préface du grand livre de E. P. Thompson, The Making of the English Working Class: "This book has a clumsy title, but it is one which meets its purpose. Making, because it is a study in an active process, which owes as much to agency as to conditioning. The working class did not rise like the sun at an appointed time. It was present at its own making ${ }^{36}$. " Dans une démarche semblable, une nouvelle histoire sociale des villes, au lieu de considérer pour donnés les groupes et pour acquise l'appartenance des individus à ces groupes qui les enferment et les définissent, tente d'inverser la perspective. Elle s'interroge sur la façon dont les relations, de niveaux multiples, qui se nouent en tous sens dans les sociétés citadines créent des solidarités, dessinent des réseaux, définissent des identités. Le vrai problème, écrit Simona Cerutti dans le livre pour le moment le plus représentatif de cette nouvelle approche, « est de comprendre comment des individus dont les histoires et les expériences sont différentes peuvent décider de se réunir et, plus encore, de se reconnaître à travers une identité sociale commune. Bref, il s'agit de s'interroger sur le rapport entre la rationalité individuelle et l'identité collective ${ }^{37}$. "

42 La liste des références possibles pour la constitution des identités citadines est longue. On peut citer, sans ordre, le sexe, le groupe d'âge, la famille, le "pays » (pour les migrants originaires d'un même lieu), le quartier, le métier, la confrérie, l'ordre, les institutions du pouvoir citadin (civil, religieux, militaire parfois), la religion, etc. Il ne s'agit pas de choisir entre ces diverses classifications pour prétendre que l'une d'entre elles (ou la combinaison de quelques-unes d'entre elles) constitue la vérité des sociétés préindustrielles. Il s'agit de les imaginer comme un ensemble de ressources qui appartiennent à l'être même de la ville à un moment et en un lieu donnés, et qui définissent l'espace d'action des citadins. Cet espace n'est évidemment pas le même pour chacun : les ressources mobilisables dépendent des lieux initiaux d'insertion sociale, des trajectoires familiales et individuelles, de la conjoncture du moment, des capacités d'interprétation de la réalité quotidienne.

Un marchand de soie immigré à Turin d'au-delà des Alpes au début du XVIII ${ }^{\mathrm{e}}$ siècle peut être membre d'une corporation de métier, d'une confrérie religieuse, de l'administration municipale, ou remplir plusieurs de ces rôles à la fois. Ses réseaux familiaux d'alliance peuvent redoubler ces premières liaisons ou au contraire en établir d'autres, sur les bases d'une complémentarité d'activité économique, d'origine ou encore de résidence. La combinaison de ses choix, imposés ou raisonnés, détermine à la fois l'importance de ses ressources, les caractères de son identité sociale, et la nature de la stratification sociale du moment fondée sur les principes alternatifs de la cohésion ou de l'homogénéité. Ainsi, ce n'est que tardivement, dans les années 1730, qu'apparaissent à Turin de nouvelles identités sociales empruntées au langage du travail et que le métier devient (alors que les premières corporations ont été fondées cent cinquante ans plus tôt) un critère décisif de stratification et de représentation sociales.

44 Contre une approche objectiviste, qui faisait des groupes sociaux des catégories "naturelles ", reconnaissables par des séries d'indicateurs transmissibles, se développe une approche plus subjective, plus individualisante, davantage intéressée par les réseaux, les stratégies, les situations, les processus que par des structures qu'on postule stables. La question, alors, est celle des modalités et des effets du recouvrement partiel, du recoupement et des exclusions des différents éléments qui forment la palette identitaire. La question est celle de leur actualisation par les acteurs sociaux dans des processus 
d'identification réciproque qui dessinent une société urbaine en constante redéfinition et qui se trouvent dessinés par elle.

Il en naît une nouvelle histoire «locale» du politique. Les institutions municipales appartiennent à la gamme des ressources inégalement mobilisables par les citadins. Si bien qu'une histoire sociale de la ville ne peut s'écrire sans prendre en compte l'histoire sociale des institutions. L'organisation institutionnelle des villes du nord de la France et $\mathrm{du}$ sud des Pays-Bas espagnols, par exemple, est d'abord une construction socioculturelle : gouvernement par les élites, autonomie locale, dispositif d'économie sociale (de «welfare town » si l'on peut dire) tout imprégné de valeurs catholiques définissent un " modèle hispano-tridentin de la bonne ville ${ }^{38}$ ». Les institutions urbaines s'inscrivent à la fin de l'Ancien Régime dans une histoire séculaire, mais c'est la conjoncture du moment qui leur donne sens et rend compte des configurations particulières qu'elles prennent dans l'espace social. Les modalités d'articulation des formes et de leurs usages ne sont pas seulement une question qui relève de l'urbanisation et de l'espace physique de la ville, mais tout autant de droit et des institutions. La contestation violente de l'ordre urbain, à son tour, doit être analysée de cette manière : non seulement comme un révélateur de comportements, de convictions, de relations, de valeurs restées autrement opaques; non seulement comme l'occasion de restituer un contexte socio-politique qui lui donne sens, elle est au contraire constitutive de son propre contexte, constitutive des identités citadines et d'un savoir social urbain ${ }^{39}$.

Invité en 1984 à dresser pour une revue italienne un panorama semblable de l'histoire urbaine, je donnais à ma conclusion une tonalité pessimiste: les risques de voir se dissoudre un objet trop complexe ne me paraissaient pas négligeables ${ }^{40}$. On aura compris, j'imagine, que je ne fais plus aujourd'hui la même analyse. Il existe des travaux qui répondent au programme vaste que j'indiquais alors: reconnaitre la diversité des formations humaines qui se succèdent derrière l'apparente permanence des sites et du vocabulaire pour contribuer à une définition historique de l'urbain. Il existe des recherches qui, au lieu de réduire la complexité de la ville, s'efforcent d'en jouer pour comprendre les processus par lesquels les acteurs redéfinissent en permanence, à la fois sans le savoir et en le voulant, l'organisation du social entendu au sens le plus large. Plus que toute autre, peut-être, l'histoire urbaine fournit l'opportunité de rompre la tautologie des descriptions en termes de catégories prédéterminées.

\section{BIBLIOGRAPHIE}

\section{Travaux récents de Bernard Lepetit sur la ville}

« Urbanization in Eighteenth Century France. A Comment », Journal of Interdisciplinary History, XXIII, 1992, p. 73-85.

avec J.-L. Pinol, « France », in R. Rodger, ed., European Urban History : Prospect and Retrospect, Leicester-Londres, Leicester University Press, 1993, p. 76-108. 
«Passé, présent et avenir des modèles urbains d'auto-organisation » et « Une herméneutique urbaine est-elle possible ? », in B. Lepetit, D. Pumain, eds, Temporalités urbaines, Paris, Anthropos, 1993, p. 113-134 et p. 287-299.

"Les temps des villes ", in Mutations économiques et urbanisation : cinq ans de recherches et d'expérimentation, Paris, La Documentation française, 1993, p. 171-196.

«Temps des formes, temps des usages », Le Courrier du CNRS, 81,1994, La Ville, p. 73-74.

« Le temps des villes », Villes, Histoire et Culture, 1, 1994, p. 7-17.

«L'appropriation de l'espace urbain : la formation de la valeur dans la ville moderne $\left(\mathrm{XVI}^{\mathrm{e}}-\mathrm{XIX}^{\mathrm{e}}\right.$ siècles) ", Histoire, Économie, Société, XIII, 1994, p. 551-559.

« Gli spazi della città », in Storia d'Europa, IV, L'età modema, Turin, Einaudi, 1995, p. 295-325.

avec C. Olmo, eds, La città e le sue storie, Turin, Einaudi, 1995.

« La ville moderne en France. Essai d'histoire immédiate », in J.-L. Biget, J.-C. Hervé, Y. Thébert, eds, Panoramas urbains. Situation de l'histoire des villes, Fontenay-St-Cloud, ENS Éditions, 1995, p. 173-207.

avec P. Clark, eds, Capital Cities and ther Hinterlands in Early Modern Europe, Aldershot, Variorum, 1996.

« Des capitales aux places centrales. Mobilité et centralité dans la pensée économique française, 1820-1850 », in Actes de la journée internationale d'Histoire urbaine, Université de São Paulo, 25 avril 1996.

\section{NOTES}

1. F. Bedarida, "The Growth of Urban History in France : some Methodological Trends ", in H. J. Dyos, ed., The Study of Urban History, Londres, E. Arnold, 1968.

2. P. Goubert, Beauvais et le Beauvaisis de 1600 à 1730, Paris, SEVPEN, 1960 ; P. Deyon, Amiens capitale provinciale. Étude sur la société urbaine au XVII siècle, Paris-La Haye, Mouton, 1967 ; M. Garden, Lyon et les Lyonnais au XVIII siècle, Paris, Les Belles Lettres, 1970 ; J.-C. Perrot, Genèse d'une ville moderne : Caen au XVIII ${ }^{e}$ siècle, Paris-La Haye, Mouton, 1975 ; J.-P. Bardet, Rouen aux XVII ${ }^{e}$ et XVIII ${ }^{e}$ siècles. Les mutations d'un espace social, Paris, SEDES, 1983 ; G. Jacquemet, Belleville au XIX $x^{e}$ siècle, du faubourg à la ville, Paris, Éditions de l'EHESS-J. Touzot, 1984. Je ne cite que les travaux qui ont une ambition d'histoire totale, développée dans un cadre monographique.

3. Quelques publications récentes: G. Livet, B. Vogler, eds, Pouvoir, ville et société en Europe, 1650-1750, Paris, Ophrys, 1983 ; M. Garden, Y. Lequin, eds, Construire la ville, XVII ${ }^{e}-X^{e}{ }^{e}$ siècles, Lyon, Presses universitaires de Lyon, 1984 ; B. Lepetit, J. Hoock, eds, La ville et l'innovation. Relais et réseaux de diffusion en Europe, XIV ${ }^{e}$-XIX ${ }^{e}$ siècles, Paris, Éditions de l'EHESS, 1987; Villes ouvrières, 1900-1950, textes réunis par S. Magri et C. Topalov, Paris, L'Harmattan, 1990 ; Les cadastres anciens des villes et leur traitement par l'informatique, édité par J.-L. Biget, J.-C. Hervé et Y. Thébert, Rome, École française de Rome, 1989.

4. La collection « Histoire des villes de France » est publiée par l'éditeur E. Privat à Toulouse ; chaque ville fait l'objet d'une monographie (une quarantaine publiées environ) menée de l'Antiquité à nos jours ; G. Duby, ed., Histoire de la France urbaine, Paris, Seuil, 1980-1985, 5 vol.

5. " Histoire et urbanisation ", Annales ESC, XXV, 1970.

6. D. Roche, « Urban History in France », in Urban History Yearbook, 1980, p. 12-22. 
7. «Ce qui vient d'être vaincu en nous, c'est précisément notre chère petite ville ", écrivait Marc Bloch en 1940 (M. Bloch, L'étrange défaite [1946], Paris, Gallimard, 1990).

8. J.-C. Perrot, op. cit., p. 553.

9. F. Choay, ed., L'urbanisme, utopies et réalités. Une anthologie, Paris, Seuil, 1965.

10. À titre d'exemple: R. Dugrand, Villes et campagnes en Languedoc. Le réseau urbain du BasLanguedoc méditerranéen, Paris, Presses universitaires de France, 1963. Pour une analyse critique : D. Pumain, T. Saint-Julien, « Fonctions et hiérarchies des villes françaises : étude du contenu des classifications réalisées en France entre 1960 et 1974 », Annales de géographie, 1976, p. 385-440

11. F. Carrière, P. Pinchemel, Le fait urbain en France, Paris, Armand Colin, 1963 ; M. Castells, La question urbaine, Paris, Maspéro, 1972.

12. En plus de M. Castells, se reporter à H. Lefebvre, La révolution urbaine, Paris, Gallimard, 1970 ; H. Lefebvre, La pensée marxiste et la ville, Paris, Casterman, 1972 ; J. Lojkine, Le marxisme, l'État et la question urbaine, Paris, Presses universitaires de France, 1977.

13. Voir par exemple F. Boudon, A. Chastel, H. Couzy, F. Hamon, Système de l'architecture urbaine, le quartier des Halles à Paris, Paris, Éditions du CNRS, 1977 ; E. Demangeon, B. Fortier, Les vaisseaux et les villes: Cherbourg, 1770-1815, Liège, Mardaga, 1978 ; J. Castex, P. Céleste, P. Panerai, Lecture d'une ville : Versailles, Paris, Éditions du Moniteur, 1980.

14. M. Foucault, Surveiller et punir, Paris, Gallimard, 1975 ; M. Foucault et alii, Les machines à guérir, aux origines de l'hôpital moderne, Paris, Institut de l'Environnement, 1976 ; A. Farge, Vivre dans la rue à Paris au XVIII siècle, Paris, Gallimard-Julliard, 1979.

15. F. Furet, L'Atelier de l'histoire, Paris, Flammarion, 1982, p. 9.

16. D. Roche, « Ville », in J. Le Goff, ed., La nouvelle histoire, Paris, Retz, 1978, p. 565-570.

17. J.-C. Perrot, « Rapports sociaux et villes au XVIII siècle », Annales ESC, XXIII, 1968, p. 241-268. On y trouve les références antérieures qui permettent de suivre le débat.

18. P. Deyon, op. cit., p. VIII-IX.

19. J.-P. Bardet, op. cit., p. 18-19.

20. L. Bergeron, M. Roncayolo, «De la ville pré-industrielle à la ville industrielle: essai sur l'historiographie française », Quaderni storici, IX, 1974, p. 827-876 ; J.-C. Perrot, op. cit.

21. A. Farge, A. Zysberg, "Les théâtres de la violence à Paris au XVIII siècle ", Annales ESC, XXXIV, 1979, p. 984-1015 ; D. Roche, Le peuple de Paris. Essai sur la culture populaire au XVIII ${ }^{e}$ siècle, Paris, Aubier, 1981 ; A. Farge, La vie fragile. Violence, pouvoirs et solidarités à Paris au XVIII siècle, Paris, Hachette, 1986.

22. J.-C. Perrot, op. cit., p. 947.

23. L. Bergeron, M. Roncayolo, op. cit., p. 827 ; F. Bedarida, "The French Approach to Urban History: an Assessment of Recent Methodological Trends ", in D. Fraser, A. Sutcliffe, eds, The Pursuit of Urban History, Londres, E. Arnold, 1983, p. 395-406.

24. B. J. L. Berry, « Cities as Systems within Systems of Cities ", Papers, Regional Science Association, 13,1964, p. 147-163.

25. B. Chevalier, Les bonnes villes de France du XIV siècle au XVIe siècle, Paris, Aubier, 1982, p. 11.

26. F. Braudel, Civilisation matérielle, économie et capitalisme, $\mathrm{XV}^{e}-\mathrm{XVII}{ }^{e}$ siècle, Paris, Armand Colin, 1979, 3 tomes (le premier tome, Les structures du quotidien, reprend en l'enrichissant un livre paru en 1967 sous un titre différent).

27. B. Lepetit, Les villes dans la France moderne (1740-1840), Paris, Albin Michel, 1988.

28. G. Arbellot, «La grande mutation des routes de France au milieu du XVIII ${ }^{\mathrm{e}}$ siècle ", Annales ESC, XXVIII, 1973, p.765-791; B. Lepetit, Chemins de terre et voies d'eau. Réseaux de transport et organisation de l'espace en France, 1740-1840, Paris, Éditions de l'EHESS, 1984 ; G. Arbellot, B. Lepetit, J. Bertrand, Atlas de la Révolution française, t. I : Routes et communications, Paris, Éditions de l'EHESS, 1987. 
29. M.-V. Ozouf-Marignier, La formation des départements. La représentation du territoire français à la fin $d u$ XVIII ${ }^{e}$ siècle, Paris, Éditions de l'EHESS, 1989.

30. B. Lepetit, "Event and Structure : the Révolution and the French Urban System », Journal of Historical Geography, XVI, 1990, p. 17-37.

31. F. Braudel, L'identité de la France, Paris, Arthaud, 1986, 3 vol.

32. Pour un exemple: W. H. Sewell Jr., Structure and Mobility: the Men and Women of Marseille, 1820-1870, Cambridge-Paris, Cambridge University Press/Éditions de la Maison des sciences de l'homme, 1985. Pour une critique, M. Gribaudi, A. Blum, «Des catégories aux liens individuels : l'analyse statistique de l'espace social», Annales ESC, XLV, 1990, p.1365-1402, et plus généralement l'ensemble de ce numéro thématique des Annales intitulé « Mobilités » (novembredécembre 1990).

33. Pour un exemple, R. Darnton, Le grand massacre des chats. Attitudes et croyances dans l'ancienne France, Paris, Robert Laffont, 1985. Pour une critique, R. Chartier, «Text, Symbols and Frenchness ", Journal of Modem History, LVII, 1985, p.682-695; et G. Levi, «I pericoli del geertzismo ", Quaderni storici, XX, 1985, p. 269-277.

34. Pour un exemple et la mise en scène d'un basculement au détriment des populations urbaines enracinées, L. Chevalier, Classes laborieuses et classes dangereuses à Paris pendant la première moitié du XIX siècle, Paris, Plon, 1958.

35. Pour une démonstration particulièrement réussie, M. Gribaudi, Itinéraires ouvriers. Espaces et groupes sociaux à Turin au début du Xx $x^{e}$ siècle, Paris, Éditions de l'EHESS, 1987.

36. E. P. Thompson, The Making of the English Working Class, Londres, V. Gollancz, 1963 (préface). Significativement, la traduction française de ce livre (Paris, Gallimard-Seuil) date de 1988.

37. S. Cerutti, La ville et les métiers. Naissance d'un langage corporatif (Turin, XVII ${ }^{e}$-XVIII ${ }^{e}$ siècle), Paris, Éditions de l'EHESS, 1990, p. 14.

38. P. Guignet, Le pouvoir dans la ville au XVIII siècle. Pratiques politiques, notabilité et éthique sociale de part et d'autre de la frontière franco-belge, Paris, Éditions de l'EHESS, 1990.

39. A. Farge, J. Revel, Logiques de la foule. L'affaire des enlèvements d'enfants, Paris 1750, Paris, Hachette, 1988; R. Descimon, «Les barricades de la Fronde parisienne, une lecture sociologique ", Annales ESC, XLV, 1990, p. 397-422.

40. B. Lepetit, «La storia urbana in Francia, scenografia di uno spazio di ricerca ", Società e Storia, VII, 1984, p. 639-666. Des éléments de la première partie de ce texte sont repris ici.

\section{RÉSUMÉS}

En France, l'histoire urbaine, depuis une vingtaine d'années, n'est pas simplement un domaine nouveau qui a connu une vive expansion. Désignée un temps comme une voie pour sortir l'histoire sociale des années soixante d'une impasse historiographique, la ville s'affirme dans les années soixante-dix comme un objet de recherche autonome, au moment où l'histoire urbaine tend à se constituer en histoire problème. Contre une approche limitée à la description des groupes sociaux, cette dernière s'intéresse désormais davantage aux réseaux, aux stratégies, aux situations, aux processus, faisant progressivement émerger la créativité des phénomènes citadins et, par la même, la ville comme un véritable sujet de l'histoire.

In France, urban history since the last twenty years is not simply a new field which has known a brisk expansion. Considered for some time as a way of extricating social history of the sixties 
from a historiographical impasse, the city affirmed itself in the seventies as an object of independent research at a moment when urban history was attempting to organize itself as a problematic history. As against an approach limited to the description of social groups, urban history henceforth concerned itself more with networks, strategies, situations or processes, gradually bringing to light the creativity of the phenomenon of town life, and at the same time the city as a true subject of history.

\section{AUTEUR}

\section{BERNARD LEPETIT}

Bernard Lepetit (EHESS) a consacré l'essentiel de ses travaux à l'histoire des villes et de l'organisation de l'espace. Il a notamment publié Chemins de terre \& voies d'eau : Réseaux de transports, organisation de l'espace, Paris, Editions de l'EHESS, 1984 ; Les villes dans la France moderne (1740-1840), Paris, Albin Michel, 1988. Il a dirigé deux volumes de l'Atlas de la Révolution française, Paris, Editions de l'EHESS, consacrés, aux Routes et communications $(1,1987)$ et à la Population $(8,1995)$ et, récemment, Les formes de l'expérience, une autre histoire sociale, Paris, Albin Michel, 1995. 